\title{
Small animal look-locker inversion recovery (SALLI)
} Daniel Messroghli ${ }^{1 *}$, Sarah Nordmeyer ${ }^{1}$, Martin Buehrer $^{2}$, Sebastian Kozerke ${ }^{2}$, Thore Dietrich ${ }^{3}$, Thomas Hucko ${ }^{3}$,
Felix Berger ${ }^{1}$, Christoph Klein ${ }^{3}$, Titus Kuehne

From 2011 SCMR/Euro CMR Joint Scientific Sessions

Nice, France. 3-6 February 2011

\section{Introduction and purpose}

Cardiac T1 mapping allows for quantitative analysis of myocardial tissue properties. Pulse sequences for human applications are not suitable for in-vivo studies in small animals. The aim of this study was to develop a single magnetic resonance imaging (MRI) approach for comprehensive assessment of cardiac function and tissue properties in small animals with high heart rates.

\section{Methods}

Small animal Look-Locker inversion recovery (SALLI) was implemented on a clinical 3 Tesla MRI system equipped with a $70 \mathrm{~mm}$ solenoid coil. SALLI combines a segmented, ECG-gated, IR-prepared Look-Locker type pulse sequence with a multimodal reconstruction framework. The pulse sequence scheme uses radial non-balanced steady-state free precession readout to minimize motion artifacts. Data acquisition can be accelerated through the use of temporal undersampling by a factor of $R$, where only a fraction of $1 / R$ profiles is acquired for each heart phase while still allowing for reconstruction of fully sampled data sets. To assess T1 accuracy in-vitro, SALLI with different sequence settings was performed in 9 agarose gel phantoms. Invivo, 10 Sprague-Dawley rats were studied to establish normal values pre- and post-injection of gadopentetate dimeglumine. One rat with surgically induced acute myocardial infarction was examined to test the potential of SALLI for the detection of acute myocardial injury.

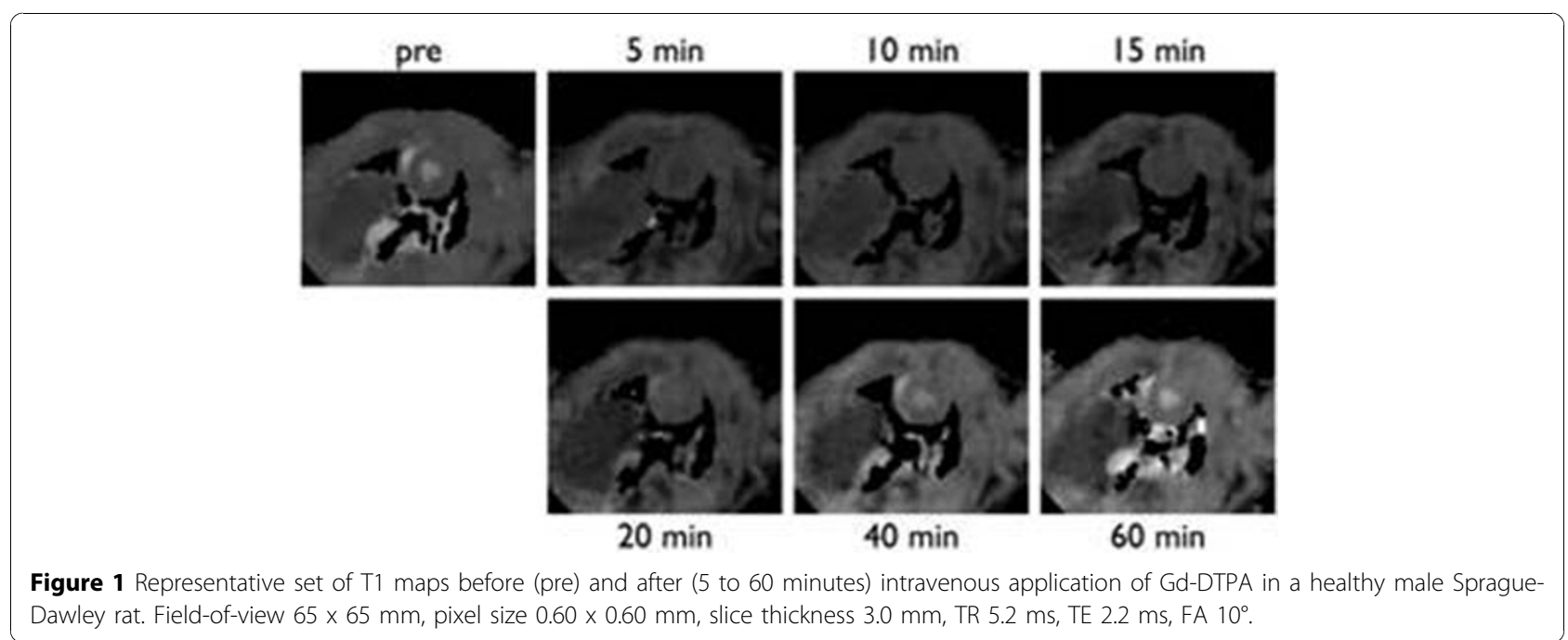

${ }^{1}$ German Heart Institute Berlin, Congenital Heart Defects and Pediatric

Cardiology, Berlin, Germany

Full list of author information is available at the end of the article

(c) 2011 Messroghli et al; licensee BioMed Central Ltd. This is an open access article distributed under the terms of the Creative 


\section{Results}

Phantom studies demonstrated systematic behavior of $\mathrm{T} 1$ measurements. In-vitro T1 error could be reduced to $1.3 \pm 7.4 \%$ using a simple linear correction algorithm. Figure 1 shows a representative set of $\mathrm{T} 1$ maps from one of the wild-type rats at different time points. Preand post-contrast $\mathrm{T} 1$ of left-ventricular myocardium and blood showed narrow normal ranges. In the animal with surgical ligation of the left circumflex artery, SALLI demonstrated hypokinesia (cine images), myocardial edema (pre-contrast T1 map), and myocardial necrosis (post-contrast T1 map and late gadolinium enhancement) in the area of infarction

\section{Conclusions}

SALLI enables simultaneous generation of cardiac T1 maps, cine, and inversion recovery (IR)-prepared images at high heart rates. T1 measurements demonstrate high accuracy in-vitro and narrow normal ranges in-vivo. SALLI might allow for comprehensive qualitative and quantitative assessment of myocardial morphology and function in small animal models of myocardial injury.

\section{Author details}

${ }^{1}$ German Heart Institute Berlin, Congenital Heart Defects and Pediatric Cardiology, Berlin, Germany. ${ }^{2}$ Institute for Biomedical Engineering, ETH and University of Zurich, Zurich, Switzerland. ${ }^{3}$ Department of Cardiology and Internal Medicine,German Heart Institute Berlin, Berlin, Germany.

Published: 2 February 2011 